\title{
Teoría de la Mente en personas con Síndrome de Asperger: estudio de caso.
}

\author{
Miriam Louro \\ Universidad de A Coruña
}

\begin{abstract}
Resumen
La Teoría de la Mente es una habilidad metacognitiva que consiste en ser capaz de ponerse en el lugar del otro, de imaginarse lo que piensa y/o siente y de comprender de manera intuitiva estados mentales, como creencias y deseos. Esta habilidad se supone deficitaria en las personas con autismo. Se presenta un estudio clínico con un niño de siete años y seis meses de edad, diagnosticado de síndrome de Asperger con el objetivo de comprobar si es capaz de resolver las tareas de falsas creencias, que se consideran miden la Teoría de la Mente.

Palabras clave: Teoría de la mente, falsas creencias, síndrome de Asperger, habilidad metacognitiva.
\end{abstract}

El síndrome de Asperger se define como un trastorno caracterizado por el mismo tipo de déficit cualitativo de la interacción social propio del autismo y por la presencia de un repertorio restringido, estereotipado y repetitivo de actividades e intereses. Difiere sin embargo del autismo en que no hay déficits o retrasos del lenguaje o del desarrollo cognoscitivo. La mayoría de los afectados son de inteligencia normal, pero suelen ser marcadamente torpes desde el punto de vista motor. Existen muchas posibilidad de que los síntomas del trastorno permanezcan en la adolescencia o en la edad adulta (García-Parajuá y Magaritos-López, 2000).

En la $5^{\circ}$ edición del DSM se incluye el síndrome de Asperger dentro de los Trastornos del Espectro del Autismo (TEA), término que hace referencia a un conjunto de trastornos cuyos síntomas, habilidades y niveles de deterioro cognitivo oscilan en cuanto al grado de afectación, desde una mayor severidad, tal es el caso del autismo clásico descrito por Leo Kanner en el 1943, hasta los síndromes más leves como el autismo de alto funcionamiento o el síndrome de Asperger (SA) (APA, 2013).

Para valorar el perfil cognitivo-conductual dentro de los TEA se plantearon diversas teorías para explicar las deficiencias en el funcionamiento cerebral de estas personas, entre las cuales cabe destacar la Teoría de la Mente. Se puede definir esta teoría como una habilidad metacognitiva que consiste en percibir el pensamiento y las sensaciones de los demás. Una persona con esta habilidad, es capaz de ponerse en el lugar del otro, de imaginarse lo que piensa y/o siente y de comprender de manera intuitiva estados mentales, como creencias y deseos. Se supone deficitaria en las personas con TEA (BaronCohen, Leslie y Frith, 1985, citado por Martín, Gómez y Garro, 2012 ).

\begin{abstract}
Método
Participantes

El sujeto participante en este estudio de caso es un niño de 7 años y 6 meses de edad que acude a terapia logopédica desde hace 4 años y medio, para trabajar la pragmática de la comunicación humana. Su diagnóstico ha variado considerablemente desde hace unos años. Primeramente le diagnosticaron un Trastorno de Déficit de Atención con Hiperactividad (TDAH), siendo informada la madre de que era un diagnóstico abierto que iba a evolucionar por la capacidad del niño. Actualmente ha sido diagnosticado de síndrome de Asperger por el equipo de valoración de ASPANAES (entidad asociativa que desarrolla su ámbito de actuación dentro de los Trastornos del Espectro de Autismo) a la espera de una revisión del mismo. Durante las sesiones se lleva a cabo una evaluación logopédica con el fin de establecer unos objetivos de intervención.
\end{abstract}

\section{Instrumentos}

Para evaluar el área del lenguaje se utilizó el Test Illinois de Aptitudes Psicolingüisticas (ITPA) (Kirk, McCarthy y Kirk, 2004). Por otro lado, con el fin de evaluar las competencias mentalistas, se utilizó el material de En la Mente (Monfort y Monfort, 2002) porque permite trabajar determinados aspectos del nivel pragmático de la comunicación humana. También fueron empleadas pruebas de falsas creencias de primer orden como la de Anne y Sally (Martín, Gómez y Garro, 2012). Finalmente, se emplearon el Inventario del Espectro Autista (IDEA) (Riviere, 2004) para valorar la severidad de los rasgos autistas, así como la Escala Australiana para el Síndrome de Asperger (ASAS) para identificar el comportamiento y las habilidades que nos pueden dar información sobre la presencia de factores indicativos de síndrome de Asperger.

\section{Procedimiento}

Se llevaron a cabo 3 sesiones de evaluación con el paciente. Las dos primeras destinadas a evaluar el lenguaje mediante el Test Illinois de Aptitudes Psicolingüísticas (Kirk, McCarthy y Kirk, 2004). Los subtest de los que consta este test son: comprensión auditiva, asociación auditiva, integración auditiva, expresión motora, comprensión visual, memoria secuencial visomotora e intregración visual. No fue posible la aplicación de los dos últimos debido al rechazo de las mismas por parte del niño. A continuación se expone una breve explicación de cada una de las pruebas aplicadas. 
Prueba de comprensión auditiva. Consiste en la narración de una historia y la posterior realización de una serie de cuestiones que el evaluado debe responder señalando a una lámina.

Prueba de asociación auditiva. Se trata de leerle al sujeto una serie de consignas, a modo de analogías, que debe completar con la palabra adecuada.

Prueba complementaria de integración auditiva. Se debe leer un listado de palabras omitiendo alguno de sus sonidos que vienen marcados por puntos suspensivos en el manual y el usuario debe repetir la palabra completa y correctamente.

Prueba de expresión motora. Consiste en enseñarle unas láminas que contienen unos determinados dibujos. Tras su visualización, el evaluado tiene que realizar un acto motor relacionado con el dibujo mostrado con anterioridad. Por ejemplo, si aparece un peine pueden realizar la acción de peinarse.

Prueba de comprensión visual. Es una prueba en la cual se le muestra una lámina con un dibujo al usuario $\mathrm{y}$, a continuación, se le enseña otra en donde tiene que señalar cuál de los dibujos que aparecen se parece al mostrado con anterioridad.

En la última sesión de evaluación se le facilitó la Escala Australiana para el Síndrome de Asperger (ASAS) a la madre, la principal responsable del cuidado del niño. Se le dio como indicación que debía cubrirla teniendo en cuenta la conducta y comportamiento que lleva a cabo su hijo, dándole un 0 a los ítems que realiza raramente y un 6 a los que realiza a menudo, pudiendo ser posibles puntuaciones intermedias. Un ejemplo de preguntas planteadas se recogen en la Tabla 1.

Tabla 1.

Ejemplo de cuestiones de la Escala Australiana para el Sindrome de Asperger (ASAS).

1.¿Carece el niño de entendimiento sobre cómo jugar con otros niños? Por ejemplo, ¿ignora las reglas no escritas sobre juego social?

2.Cuando tiene libertad para jugar con otros niños, como en el recreo, ¿evita el contacto social con los demás ¿ Por ejemplo, busca un lugar retirado o se va a la biblioteca.

3.Parece el niño ignorar las convenciones sociales o los códigos de conducta, y realiza acciones o comentarios inapropiados? Por ejemplo, un comentario personal sin ser consciente de que puede ofender a los demás.

4.¿Carece el niño de empatía, de entendimiento intuitivo de los sentimientos de otras personas? Por ejemplo, no darse cuenta de que una disculpa ayudará a la otra persona a sentirse mejor.
Esta escala nos da información para establecer una serie de objetivos terapéuticos, sobre todo nos indica que sería apropiado la intervención en el área de la pragmática, motivo por el cual el niño acude a terapia logopédica.

En relación al Inventario Del Espectro Autista (IDEA), se ha cubierto a lo largo de las sesiones de valoración. En el apartado de resultados se muestran las dimensiones evaluadas junto con la puntuación obtenida en cada una de ellas. En la Tabla 2 se muestran, a modo de ejemplo, los ítems de la dimensión social que tiene en cuenta el IDEA, concretamente el apartado de relaciones sociales.

Tabla 2.

Dimensión social del IDEA

1. Relaciones sociales

Aislamiento completo. No apego a personas específicas. No relación con adultos o iguales.

Incapacidad de relación. Vínculos con adultos. No relación con iguales.

Relaciones infrecuentes, inducidas, externas con iguales. Las relaciones más como respuestas que como iniciativa propia. Motivación de relación con iguales, pero falta de relaciones por dificultad para comprender sutilezas sociales y escasa empatía. Conciencia de soledad.

No hay trastorno cualitativo de la relación.

\section{Tratamiento}

Se llevó a cabo la terapia logopédica en un gabinete, en el cual se desarrolló el Prácticum II de la autora del trabajo, durante una sesión semanal e individual de 45 minutos.

Para trabajar los aspectos pragmáticos (especialmente, teoría de la mente) se utilizó como material de referencia el manual En la Mente (Monfort y Monfort, 2002) durante un período de 6 sesiones. Un relevante objetivo terapéutico consistía en que fuese capaz de aprender a solucionar tareas de falsas creencias.

Se comenzó a trabajar con una prueba de falsa creencia de $1^{\mathrm{o}}$ orden, más concretamente, la tarea consistía en presentarle la lámina $\mathrm{n}^{\circ} 54$ del manual de En la Mente mostrada a continuación. La pregunta que se le realiza al paciente tras contemplar la lámina sería ¿qué crees que pensaría el señor?, o también ¿qué pensaría el señor que ha pasado?. La respuesta por parte del niño fue correcta. 


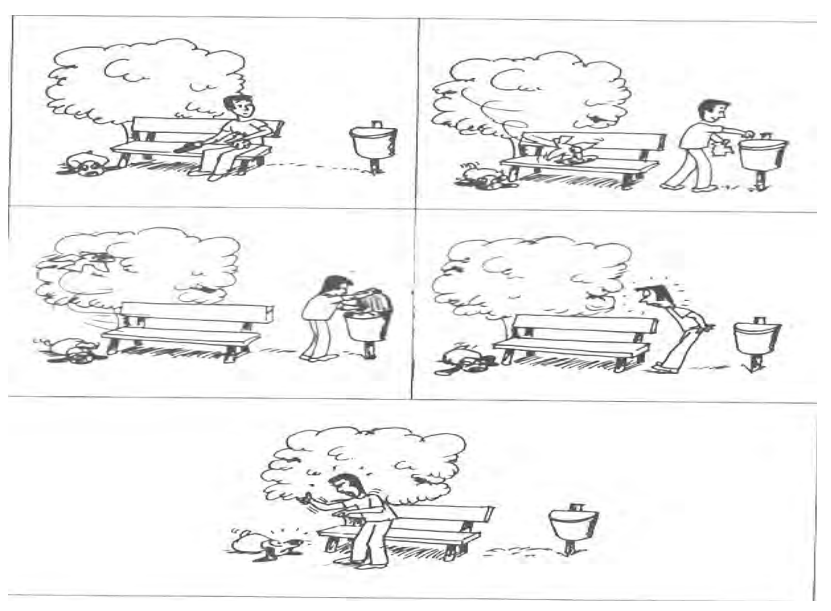

Figura 1: Lámina ${ }^{\circ} 54$ del manual de En la Mente.

A pesar de que la anterior tarea la realizó correctamente, se utilizó también otra prueba de falsa creencia de $1^{\circ}$ orden, concretamente la recreación de la prueba de Anne y Sally. Esta tarea consiste en contarle la siguiente situación de las dos muñecas:"Sally tiene una cesta y Anne una caja. Sally pone una canica en la cesta y se marcha. Mientras Sally está fuera, Anne va a la cesta, coge la canica y la coloca en su propia caja. Finalmente vuelve Sally" y se pregunta al paciente "¿en dónde buscará Sally su canica?". Se le aportaron una serie de apoyos debido a las dificultades que presentó el niño a la hora de dar una respuesta. Los apoyos prestados fueron los siguientes: (a) se narró la historia aumentando la entonación en las partes de mayor importancia, (b) le ofrecimos la historia visualmente en una lámina, (c) se repitió la historia las veces que fueron necesarias para su comprensión y (d) se representó la secuencia con objetos reales.

También se trabajaron las pruebas de falsas creencias de $2^{\circ}$ orden, concretamente con la lámina $\mathrm{n}^{\circ} 59$ del manual de En la Mente. Para la realización de esta tarea de falsas creencias, tras mostrarle la lámina se le preguntó al niño: (a) ¿qué creerán los niños que hay en la caja? y (b) ¿por qué pensará el padre que se mueve la caja?.

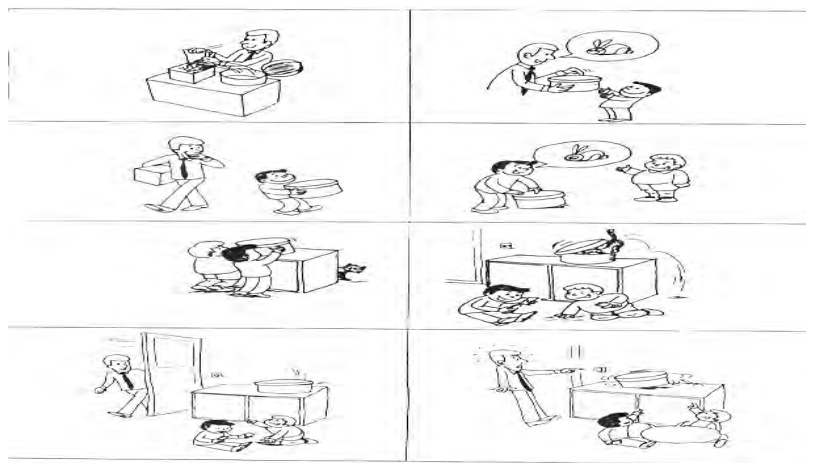

Figura 2: Lámina n ${ }^{\circ} 59$ del manual de En la Mente.
A continuación se trabajaron las bromas con la lámina $n^{\circ} 75$ del manual. En ella aparecen dos viñetas diferentes. En la primera se le preguntó (a) ¿qué le diría el niño que está señalando la cabeza de su amigo?, (b) darle el inicio de la frase para evitar ese bloqueo inicial de no saber qué responder, concretamente se le dijo: "tienes...". En la siguiente viñeta se le dio la siguiente ayuda verbal: (a) la niña lo avisa de que hay algo peligroso en el agua".

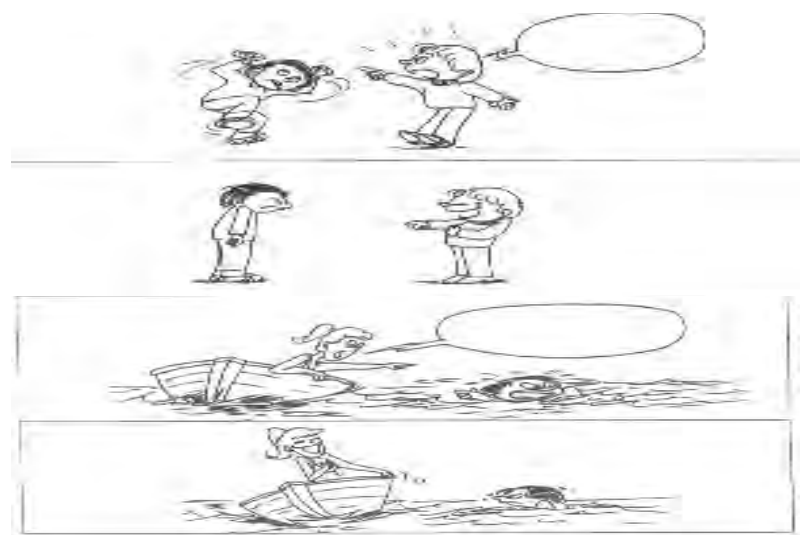

Figura 3: Lámina $n^{\circ} 75$ del manual de En la Mente.

\section{Resultados}

En el Inventario de Espectro Autista (IDEA), como puede observarse en la Tabla 3, el sujeto obtuvo una puntuación total de 15 . Las puntuaciones en torno a 24 puntos son propias de los cuadros clínicos del síndrome de Asperger, mientras que las situadas en torno a 50 puntos serían propias del Autismo de Kanner. Como la puntuación total fue de 15 se situaría en el primer caso. Esto no es un diagnóstico clínico, pero ayuda a comprobar en qué dimensiones se debe de incidir más. Además, ayuda a conocer el grado de severidad del Trastorno del Espectro del Autismo.

Tabla 3 .

Resultado del IDEA por dimensiones.

\begin{tabular}{l|c}
\hline Dimensión social. & \multicolumn{2}{|l}{ Puntuacio } \\
nes
\end{tabular}




\begin{tabular}{l|c}
\hline \hline Dimensión de la socialización. & \\
\hline Trastorno de la ficción e imaginación. & 0 \\
Trastorno de la imitación. & 0 \\
Trastorno de la suspensión. & 2 \\
\hline Total & 15 \\
\hline
\end{tabular}

En cuanto a los resultados obtenidos en el ITPA no se pudo obtener un perfil de aptitudes completo, al haber resultado imposible la aplicación de todas las áreas recogidas en este instrumento. En la Tabla 4 podemos observar las puntuaciones directas (PD) y típicas (PT) obtenidas en cada subtest junto con la edad psicolingüística que le corresponde.

Tabla 4.

Resultados de los subtests del ITPA.

\begin{tabular}{lcc}
\hline Subtest & $\begin{array}{c}\text { Puntuación } \\
\text { directa (PD) }\end{array}$ & $\begin{array}{c}\text { Puntuación } \\
\text { típica (PT) }\end{array}$ \\
\hline Comprensión auditiva & 32 & 35 \\
Comprensión visual & 27 & 40 \\
Asociación auditiva & 21 & 30 \\
Expresión motora & 23 & 53 \\
Test complementario de & 20 & 57 \\
integración auditiva & & \\
\hline
\end{tabular}

La información útil es el análisis de discrepancias que se realiza utilizando la puntuación típica obtenida y la media (suma de todas las puntuaciones directas dividida entre 10 que es el número de subtests). Si la puntuación típica es inferior a la media, necesitaría mejorar en esas áreas. Por lo contrario si es superior a la media, estaría en un nivel adecuado, son los puntos fuertes y puntos débiles; el conocimiento de los mismos resultó de ayuda a la hora de formular los objetivos de intervención. En la Tabla 5 se pueden apreciar los puntos con peores resultados: apartados de Comprensión Auditiva y Asociación Auditiva, mientras que sus puntos fuertes fueron la Comprensión Visual y la Expresión Motora.

Finalmente, con respecto a la pragmática de la comunicación y, en este caso, a las tareas mentalistas se pudo comprobar que mediante el entrenamiento, de diversas pruebas de falsas creencias de $1^{\circ}$ orden, fue capaz de realizar correctamente, con apoyo, tareas que antes no realizaba como la prueba de Sally y Anne. Como podemos observar en la Tabla 10, en un primer momento preintervención, el niño daba respuestas
Tabla 5 .

Análisis de Discrepancias del ITPA.

\begin{tabular}{lcccc}
\hline Subtest & $\begin{array}{c}\text { Puntuación } \\
\text { típica (PT) }\end{array}$ & Media & $\begin{array}{c}\text { Puntos } \\
\text { fuertes }\end{array}$ & $\begin{array}{c}\text { Puntos } \\
\text { débile } \\
\mathrm{s}\end{array}$ \\
\hline $\begin{array}{l}\text { Comprensión } \\
\text { auditiva }\end{array}$ & 35 & 39.5 & - & -4.5 \\
$\begin{array}{l}\text { Asociación } \\
\text { auditiva }\end{array}$ & 30 & 39.5 & - & -9.5 \\
$\begin{array}{l}\text { Expresión } \\
\text { verbal }\end{array}$ & - & 39.5 & - & - \\
$\begin{array}{l}\text { Comprensión } \\
\text { visual }\end{array}$ & 40 & 39.5 & 0.5 & - \\
$\begin{array}{l}\text { Asociación } \\
\text { visual }\end{array}$ & - & 39.5 & - & - \\
$\begin{array}{l}\text { Expresión } \\
\text { motora }\end{array}$ & 53 & 39.5 & 13.5 & - \\
\hline
\end{tabular}

erróneas a esta recreación, indicando que Sally buscaría su canica en la caja. Tras aportarle diversas ayudas, resolvió correctamente la tarea mentalista. No ocurrió lo mismo con las tareas de falsas creencias de $2^{\circ}$ orden, en las cuales sigue cometiendo errores en la resolución de las mismas. Concretamente en la lámina $n^{\circ} 59$, en la cual el padre se asusta al ver que la caja, que tendría que estar vacía, se está moviendo porque se había metido un gato al que nadie había visto. La respuesta por parte del niño fue: "Los niños saben que hay un gato dentro de la caja. El padre tenía mucho miedo. Por tanto, la respuesta dada fue errónea.

Cabe decir que al principio no resolvía, por sí solo, tareas sobre bromas, bloqueándose al inicio e intentado cambiar de tema, por lo cual se le dieron las ayudas detallas con anterioridad. Tras aportarle esas ayudas, fue capaz de resolver las tareas correctamente aunque con un patrón monótono de habla. Esto se puede comprobar concretamente en la lámina $\mathrm{n}^{\circ} 75$ del manual a la que respondió, tras darle apoyos verbales: "Tienes algo en la cabeza, el niño se asusta, el otro se ríe mucho, ja ja es una broma". Esta evocación presentó un tono monótono de habla que podría mostrar el aprendizaje por repetición de este tipo de tareas, así como la anterior realización de la misma. En la siguiente viñeta, en la cual aparece una niña señalando algo y un niño gritando que a continuación pone cara de enfado, no hubo respuesta oral por parte del paciente. Debido a ésto, se le dio como ayuda verbal: (a)la niña lo avisa de algo 
peligroso". Entonces el niño respondió: "Hay un tiburón o una ballena y él se asusta pero era una broma, jaja". Se pudo apreciar también un tono monótono en la evocación de la respuesta.

Tabla 6.

Comparación entre los resultados pre y post intervención

\begin{tabular}{lcc}
\hline Tarea & $\begin{array}{c}\text { Pre } \\
\text { intervención }\end{array}$ & $\begin{array}{c}\text { Post } \\
\text { intervención }\end{array}$ \\
\hline $\begin{array}{l}\text { Falsas creencias de } 1^{\circ} \\
\text { orden }\end{array}$ & $\mathrm{Si}$ & $\mathrm{Si}$ \\
$\begin{array}{l}\text { Falsa creencia de } 1^{\circ} \\
\text { orden: Anne y Sally }\end{array}$ & $\mathrm{No}$ & $\mathrm{Si}$ \\
$\begin{array}{l}\text { Falsas creencias de } 2^{\circ} \\
\text { orden }\end{array}$ & $\mathrm{No}$ & $\mathrm{No}$ \\
Bromas & $\mathrm{No}$ & $\mathrm{Si}$ \\
\hline
\end{tabular}

\section{Discusión y conclusiones}

En el presente estudio de caso se han utilizado diversas tareas para evaluar y entrenar la Teoría de la Mente como son las falsas creencias de $1^{\circ}$ y $2^{\circ}$ orden, las bromas y la recreación de la tarea de Anne y Sally. El niño evaluado daba respuestas correctas a algunas tareas de falsas creencias de $1^{\circ}$ orden, fracasando en la recreación de la prueba de Anne y Sally, en las falsas creencias de $2^{\circ}$ orden y en tareas sobre bromas. Durante el proceso de intervención se consiguió, con la aportación de ayudas, superar las tareas de falsas creencias de $1^{\circ}$ orden en general y más concretamente la recreación de las muñecas Anne y Sally. También se consiguió el aprendizaje de bromas, el cual se tiene que seguir trabajando para evitar la elaboración de respuestas monótonas. Finalmente, no fue posible un aprendizaje de las falsas creencias de $2^{\circ}$ orden, en las cuáles fue necesario aportar numerosos apoyos sin obtener una respuesta correcta.

Tales resultados ponen de manifiesto que el entrenamiento formal y explícito de las tareas mentalistas y otras habilidades pragmáticas, permiten mejorar significativamente, desde un punto de vista clínico, las tareas en las que previamente había fracasado nuestro paciente, en este caso mediante una serie de apoyos. Para afianzar estos aprendizajes, es necesario, además de continuar con el tratamiento llevado a cabo hasta ahora, generalizarlos a los ambientes cotidianos del niño (casa, parque, colegio, etc.) así como, ante diferentes personas (familiares, profesores, amigos, etc.) e ir reduciendo progresivamente los apoyos prestados hasta conseguir las metas propuestas. Es relevante tener en consideración el grado de dificultad de las diversas tareas puesto que no todas tienen la misma dificultad, ya que requieren diferentes niveles de ayuda para entrenarlas. Suelen ser más fáciles de entrenar las pruebas más cercanas a la vida social y educativa del niño (Gómez et al., 2007).

Por tanto, nuestro estudio de caso permite concluir que el entrenamiento llevado a cabo, en este caso con la aportación de los apoyos necesarios, ha mejorado las habilidades metacognitivas de nuestro paciente, coincidiendo de esta manera con estudios previos, en los cuales se puede comprobar la mejoría del sujeto tras someterse a un entrenamiento formal y explícito de las pruebas de falsas creencias (Martín, Gómez y Garro, 2012).

\section{Referencias \\ APA (2013). Guía de consulta de los criterios diagnósticos del DSM 5. Arlington: Asociación Americana de Psiquiatría.}

Galve, J. L. y Ayala, C. L. (2001). Cuestionario de Antecedentes Personales (CAP). Evaluación $e$ informes psicopedagógicos. Madrid: CEPE.

García Parajuá, P. y Magaritos López, M. (2000). Guía de bolsillo de la clasificación CIE-10: clasificación de los trastornos mentales y del comportamiento. Madrid: Médica Panamericana S.A.

Gómez, I., Martín, M. J., Chávez, M. y Greer, D. (2007). Perspective taking in children with autism. European Journal of Behavior Analysis, 8,13-28. 30.

Kirk, S. A., McCarthy, J. J. y Kirk, W. D. (2004). Test de Aptitudes Psicolingüísticas (ITPA). Madrid: TEA Ediciones.

Martín García, M. J., Gómez Becerra, I. y Garro Espín, M. J. (2012). Teoría de la Mente en un caso de autismo: ¿cómo entrenarla?. Psicothema, 24, 542547.

Monfort, M. y Monfort, I. (2002). En la mente: Un soporte gráfico para el entrenamiento de las habilidades pragmáticas en niños. Madrid: Entha Ediciones.

Raven, J. C., Court, J. H. y Raven, J. (1996). Raven Matrices Progresivas, Manual. Madrid: TEA Ediciones S.A.

Riviere A. (2004). IDEA: Inventario del Espectro Autista. Buenos Aires: FUNDEC. 